\title{
LA AGENDA NACIONAL DE INVESTIGACIÓN EN RECURSOS HUMANOS EN SALUD: GENERANDO EVIDENCIAS PARA MEJORAR LAS COMPETENCIAS EN EL SECTOR SALUD
}

\author{
[NATIONAL AGENDA FOR RESEARCH IN HUMAN RESOURCES FOR \\ HEALTH: GENERATING EVIDENCE FOR IMPROVING THE HEALTH \\ SECTOR COMPETENCES]
}

\author{
Martín Yagui 1,2,a,b, Javier Vargas ${ }^{1,2, b}$
}

La Organización Panamericana de la Salud y el Organismo Regional Andino de Salud reconocen que, en los últimos años, los países de la sub región andina han venido mostrando una creciente preocupación por el tema de los Recursos Humanos en Salud (RHUS), haciéndose esto visible en sus respectivas agendas ${ }^{(1)}$. De acuerdo con su análisis, los países de la región, enfrentan problemas de accesibilidad, cobertura y calidad de los servicios que se asocian con una inequidad en la disponibilidad y distribución de profesionales y técnicos de salud, así como, con la falta de adecuación de su formación a las necesidades sanitarias de la población.

Por su parte, el Ministerio de Salud identificó cinco grupos de problemas en el campo de los RHUS: la desarticulación entre la formación y el campo laboral, la ausencia de rectoría en el campo de los RHUS, la precarización del empleo, la exigencia de mayor rendimiento del trabajador, y el interés creciente del personal por su capacitación, en especial en el nivel del post grado ${ }^{(2)}$. La atención de estos problemas, incluyendo, la verificación misma de su situación problemática, requiere de la producción de evidencias que permitan su caracterización y explicación para facilitar el proceso de toma de decisiones de todas las instancias involucradas.

Atendiendo a la necesidad de mejorar la eficiencia en la asignación de recursos públicos para la ejecución de actividades de investigación en salud, el Instituto Nacional de Salud organizó y condujo el proceso de identificación de las prioridades nacionales de investigación en salud, Perú 2010 -2014 ${ }^{(3)}$ y como resultado de dicho proceso se identificó como primera prioridad nacional a las "investigaciones para conocer los problemas de los recursos humanos en salud". Tomando en cuenta que este resultado se obtuvo con el concurso de más de mil representantes del sector salud de todo el país, quienes participaron en 20 talleres regionales, un taller de análisis del Plan Nacional Concertado de Salud y finalmente un foro nacional, podemos afirmar que este resultado expresa un consenso de los actores que trabajan tanto en los ámbitos público como privado, regional como nacional, académico como ejecutivo del sector, y que perciben que existen brechas en el conocimiento que involucra a los RHUS, que necesitan ser cubiertas para apoyar el proceso de toma de decisiones a favor de mejorar la gerencia de los servicios de salud.

Luego de la identificación de las Prioridades Nacionales de Investigación en Salud, se procedió a la identificación de la Agenda Nacional o líneas de Investigación en RHUS, para ello se buscó responder a la siguiente interrogante: ¿Qué es lo que se requiere investigar a fin de lograr entender y solucionar la problemática de recursos humanos en salud y mejorar el desempeño del sistema de salud? La agenda nacional de investigación en recursos humanos ha definido treinta temas, que abordan desde la necesidad de articular la formación en pregrado con las necesidades sanitarias o la utilización de las tecnologías de información y comunicación en el proceso de enseñanza-aprendizaje, hasta el marco normativo vinculado a los RHUS, pasando por la evaluación del impacto económico y sanitario de la migración interna y externa de profesio-

\footnotetext{
Instituto Nacional de Salud. Lima, Perú.

2 Universidad Nacional Mayor de San Marcos. Lima, Perú.

a Médico Patólogo Clínico; ${ }^{\text {b } M e ́ d i c o ~ E p i d e m i o ́ l o g o . ~}$
} 
nales y los factores asociados a su movilización, competencias docentes, necesidades formativas en el ámbito regional, la evaluación del impacto de las experiencias de especialización y formación en el post grado o la formación de agentes comunitarios, los retos que plantea el aseguramiento universal, etc.

En el presente número de la Revista Peruana de Medicina Experimental y Salud Pública, además de presentar la agenda de los temas de investigación relacionados a los RHUS, se presenta el proceso de construcción social que ha seguido su elaboración ${ }^{(4)}$, porque consideramos que también se constituye en una oportunidad de aprendizaje en la perspectiva de fortalecer el desempeño del sistema de salud y de servir como referente nacional y regional para procesos análogos. La agenda de investigación en RHUS se propone como un eje articulador de las iniciativas de investigación y de la canalización de recursos públicos y privados de temas que estarán en la agenda sanitaria nacional en los siguientes años, tales como la descentralización de los servicios, el aseguramiento universal, el cumplimiento de los objetivos del milenio, etc., y el proceso de su elaboración, plantea desafíos para construir redes que faciliten el desarrollo de competencias en investigación y la gestión de la investigación en RHUS y que vinculen a los niveles locales y regionales como los establecimientos de salud y las Direcciones Regionales de Salud con los niveles nacionales como la Oficina General de Recursos Hu- manos del MINSA o el Consejo Nacional de Ciencia y Tecnología.

Creemos que la priorización de la investigación en RHUS es el inicio de un proceso de generación de conocimiento sobre la administración de los servicios de salud en todos sus niveles, enfatizando en el papel que juegan las personas en su dinámica, lo cual que debe rendir sus frutos en el proceso de toma de decisiones basadas en la evidencia sobre los RHUS.

\section{REFERENCIAS BIBLIOGRÁFICAS}

1. Organización Panamericana de la Salud. Plan Andino de Recursos Humanos en Salud. REMSAA XXIX/449. Bogotá: OPS; 2008.

2. Instituto de Desarrollo de Recursos Humanos en Salud. El Perú respondiendo a los desafíos de la década de los Recursos Humanos en Salud. $1^{\text {ra }}$ edición. Serie Recursos Humanos en Salud No. 05, Lima: MINSA; 2006.

3. Caballero $P$, Yagui M, Espinoza M, Castilla T, Granados A, Velásquez A, et al. Prioridades Regionales y Nacionales de Investigación en Salud, Perú 2010 - 2014: Un proceso con enfoque participativo y descentralista. Rev Peru Med Exp Salud Pública. 2010; 27(3):398-410.

4. Curisinche M, Yagui M, Castilla T, Cabezas C, Escalante $\mathbf{G}$, Casas $\mathbf{M}$ et al. Proceso de construcción de la agenda nacional de investigación sobre recursos humanos en salud (RHUS) en el Perú, 2011-2014. Rev Peru Med Exp Salud Publica. 2011;28(2):372-81.

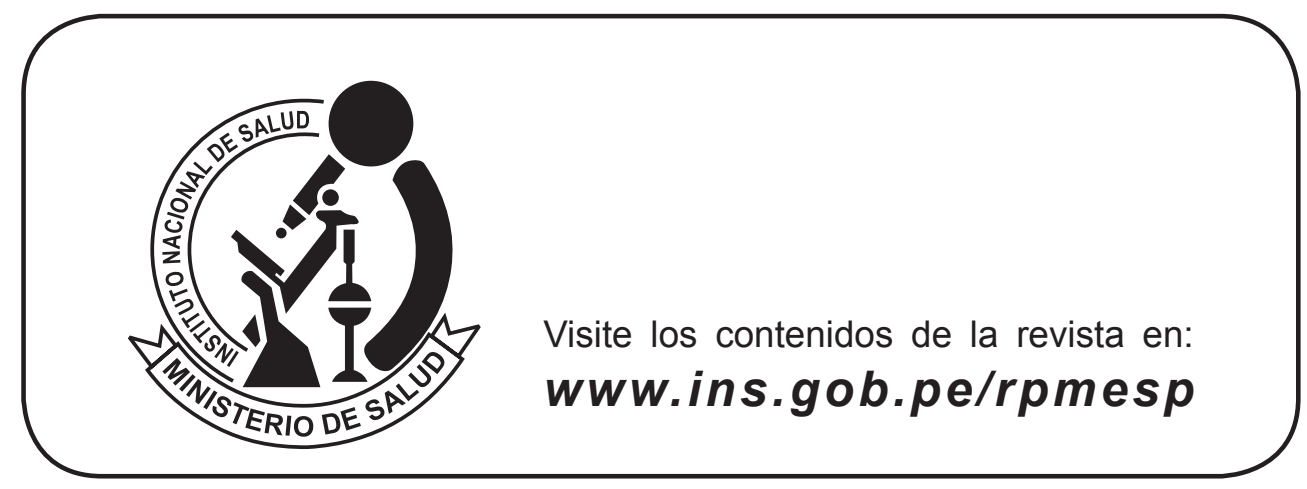

